\title{
On Complex Lee and Yang Polynomials
}

\author{
Bernard Beauzamy \\ Institut de Calcul Mathématique, 37 rue Tournefort, 75005 Paris, France. \\ E-mail: beauzamy@mathp7.jussieu.fr
}

Received: 8 September 1995/Accepted: 5 April 1996

\begin{abstract}
We introduce a family of polynomials $P_{n}\left(z_{1}, \ldots, z_{n}\right)$ with complex coefficients, having the same property as the real Lee and Yang polynomials: the zeros of $P_{n}(z, \ldots, z)$ all lie on the unit circle. We deduce from this construction a class of inner functions in several complex variables.
\end{abstract}

In the book by D. Ruelle "Statistical Mechanics" [2], Ch. 5 (The Problem of Phase Transitions) begins with the study of a family of polynomials $P_{n}\left(z_{1}, \ldots, z_{n}\right)$, introduced by Lee and Yang in 1952 (see also the article [3] by Ruelle for an update). They have the remarkable property that, for all $n$, the associated one-variable polynomial $P_{n}(z)=P_{n}(z, \ldots, z)$ has all its roots on the unit circle. This property of the zeros of $P_{n}(z)$ is deduced from the following assertion on $P_{n}\left(z_{1}, \ldots, z_{n}\right)$ :

$$
\text { if } P_{n}\left(z_{1}, \ldots, z_{n}\right)=0 \text { and if }\left|z_{1}\right| \leqq 1, \ldots,\left|z_{n-1}\right| \leqq 1, \quad \text { then }\left|z_{n}\right| \geqq 1 .
$$

Two comments are in order:

- The way (1) is proved by Lee and Yang is by means of complex analysis, and more precisely by means of "bilinear maps" of the form $z \rightarrow(a z+b) /(c z+d)$; the whole proof looks quite similar to the one given by J.L. Walsh for the socalled "Walsh's contraction principle" (see J.L. Walsh [4], B. Beauzamy [1]). This is also the case for more recent proofs, such as Asano's (see [3]).

- No direct proof is known (at least to me) of the fact that the $P_{n}(z)$ 's have their roots on the unit circle (except in a special case, see below). Apparently, one "has" to go through the "decoupling procedure" $P_{n}\left(z_{1}, \ldots, z_{n}\right)$ in order to ensure this.

We say that we have a decoupling procedure if we can replace a polynomial $P_{n}(z)$ in one variable by a polynomial $P\left(z_{1}, \ldots, z_{n}\right)$, linear in each $z_{j}$ (not necessarily symmetric), with the property that $P(z, \ldots, z)=P(z)$. The author gave in [1]

Supported by contract E.T.C.A./C.R.E.A. 20461/95 (Ministry of Defense, France) and by NATO grant CRG 930760 
an example of a symmetric decoupling procedure, using Walsh's contraction principle, which allows the construction of an algorithm finding the zeros of a complex one-variable polynomial. Here, the $P_{n}\left(z_{1}, \ldots, z_{n}\right)$ appear as another (non-symmetric) decoupling procedure of the $P_{n}(z)$. Since these decoupling procedures appear to be very efficient tools for the study of one-variable polynomials, it is quite important to understand them in their full strength: how do they work? To what polynomials do they apply?

The polynomials introduced by Lee and Yang have one "drawback" (connected with their physical interpretation): they have real coefficients, though the methods of study are purely of complex nature. So clearly the study of a general decoupling procedure based upon these ideas can be made only within the proper frame, which is that of complex polynomials, and this is what we do here, so as to eliminate all "artefacts" connected with real numbers.

In some sense, we provide a partial answer to the question raised by David Ruelle in [3]: why is the Lee-Yang theorem an isolated result? We view it, here, as one element in the general frame - still to be constructed - of the decoupling procedures.

I thank Enrico Bombieri for pointing out the reference Ruelle [2] and its possible connection with Walsh's principle.

\section{The Real Lee and Yang Polynomials}

We use the notation from Ruelle. Let $\left(A_{i, j}\right)_{i \neq j}$ be a family of real numbers such that $-1 \leqq A_{i, j} \leqq 1, A_{i, j}=A_{j, i}$, for $i=1, \ldots, n, j=1, \ldots, n$. We define

$$
P_{n}\left(z_{1}, \ldots, z_{n}\right)=\sum_{S} z^{S} \prod_{i \in S} \prod_{j \in S^{\prime}} A_{i, j}
$$

where the summation is over all subsets $S=\left\{i_{1}, \ldots, i_{S}\right\}$ of $1, \ldots, n, z^{S}=z_{i_{1}} \cdots z_{i_{s}}$, and $S^{\prime}=\left\{j_{1}, \ldots, j_{n-s}\right\}$ is the complement of $S$ in $1, \ldots, n$. If $S$ or $S^{\prime}$ is empty, the corresponding coefficient is assumed to be 1 .

Thus we have

$$
\begin{aligned}
P_{1}\left(z_{1}\right)= & 1+z_{1}, \\
P_{2}\left(z_{1}, z_{2}\right)= & 1+A_{1,2} z_{1}+A_{2,1} z_{2}+z_{1} z_{2}, \\
P_{3}\left(z_{1}, z_{2}, z_{3}\right)= & 1+A_{1,2} A_{1,3} z_{1}+A_{2,1} A_{2,3} z_{2}+A_{3,1} A_{3,2} z_{3} \\
& +A_{1,3} A_{2,3} z_{1} z_{2}+A_{1,2} A_{3,2} z_{1} z_{3}+A_{2,1} A_{3,1} z_{2} z_{3}+z_{1} z_{2} z_{3} .
\end{aligned}
$$

A first remark is that, for $n \geqq 3$, the polynomials $P_{n}\left(z_{1}, \ldots, z_{n}\right)$ are not symmetric. If we set $A_{1,2}=A_{2,1}=a, A_{1,3}=A_{3,1}=b, A_{2,3}=A_{3,2}=c$, then $P_{3}(1,0,0)=1+$ $a b, P_{3}(0,1,0)=1+a c$.

A second remark is that the statement " $P_{n}(z)$ has all its roots on the unit circle" is wrong in general if we do not assume all the coefficients to be real. Indeed, consider $P_{2}\left(z_{1}, z_{2}\right)=1+i z_{1}+i z_{2}+z_{1} z_{2}$, then $P_{2}(z)=1+2 i z+z^{2}$, which does not have its roots on the unit circle. The fact that all coefficients need to be real for the proof to work is well-hidden in [2]! 
The polynomials $P_{n}\left(z_{1}, \ldots, z_{n}\right)$ satisfy the following relation, easily deduced from the definition:

$$
\begin{aligned}
P_{n}\left(z_{1}, \ldots, z_{n}\right)= & z_{n} A_{n, 1} \cdots A_{n, n-1} P_{n-1}\left(z_{1} / A_{n, 1}, \ldots, z_{n-1} / A_{n, n-1}\right) \\
& +P_{n-1}\left(A_{n, 1} z_{1}, \ldots, A_{n, n-1} z_{n-1}\right)
\end{aligned}
$$

and they are "reciprocal", in the sense that

$$
P_{n}\left(1 / z_{1}, \ldots, 1 / z_{n}\right)=\frac{1}{z_{1} \cdots z_{n}} P_{n}\left(z_{1}, \ldots, z_{n}\right)
$$

We now turn to the complex definition.

\section{The Complex Lee and Yang Polynomials}

Our starting point will not be an intrinsic definition such as (2), but an induction relation of the type (3). We observe indeed that $P_{n}\left(z_{1}, \ldots, z_{n}\right)$ is affine in each variable, so the equation $P_{n}=0$ is equivalent to a relation of the form $z_{n}=\left(A z_{n-1}+\right.$ $B) /\left(C z_{n-1}+D\right)$, where $A, B, C, D$ depend only on $z_{1}, \ldots, z_{n-2}$.

Lemma 1. An expression of the form

$$
\varphi(z)=\frac{A z+B}{C z+D}
$$

satisfies $|\varphi(z)|=1$ for all $z,|z|=1$, if and only if it can be written

$$
\varphi(z)=\kappa \frac{a z+b}{\bar{b} z+\bar{a}},
$$

for some complex numbers $a, b, \kappa$, with $|\kappa|=1$.

This lemma is obvious.

Proposition 2. Assume we have a family of polynomials $P_{n}\left(z_{1}, \ldots, z_{n}\right)$, with complex coefficients, satisfying the following induction hypothesis:

$$
\begin{aligned}
P_{1}\left(z_{1}\right)= & A_{1} z_{1}+\overline{A_{1}}, \\
P_{n}\left(z_{1}, \ldots, z_{n}\right)= & z_{n} A_{n, 1} \cdots A_{n, n-1} P_{n-1}\left(z_{1} / A_{n, 1}, \ldots, z_{n-1} / A_{n, n-1}\right) \\
& +P_{n-1}\left(\overline{A_{n, 1}} z_{1}, \ldots, \overline{A_{n, n-1}} z_{n-1}\right) .
\end{aligned}
$$

Then the conditions

$$
\left|z_{1}\right|=\cdots=\left|z_{n-1}\right|=1 \text { and } P_{n}\left(z_{1}, \ldots, z_{n}\right)=0
$$

imply $\left|z_{n}\right|=1$.

(In this proposition, there is no restriction on the $A_{i, j}$ 's.) 
Proof. We write $\alpha_{j}=A_{n, j}, \beta_{j}=A_{n-1, j}, A=\alpha_{1} \cdots \alpha_{n-2}, B=\beta_{1} \cdots \beta_{n-2}$. We have

$$
P_{n-1}\left(z_{1}, \ldots, z_{n-1}\right)=z_{n-1} B P_{n-2}\left(z_{1} / \beta_{1}, \ldots, z_{n-2} / \beta_{n-2}\right)+P_{n-2}\left(\overline{\beta_{1}} z_{1}, \ldots, \overline{\beta_{n-2}} z_{n-2}\right) .
$$

If $P_{n}\left(z_{1}, \ldots, z_{n}\right)=0$, we get, substituting in (5):

$$
-z_{n}=\frac{\overline{\alpha_{n-1}} z_{n-1} B P_{n-2}\left(\frac{\overline{\alpha_{1} z_{1}}}{\beta_{1}}, \ldots, \frac{\overline{\alpha_{n-2} z_{n-2}}}{\beta_{n-2}}\right)+P_{n-2}\left(\overline{\alpha_{1}} \overline{\beta_{1}} z_{1}, \ldots, \overline{\alpha_{n-2}} \overline{\beta_{n-2}} z_{n-2}\right)}{A B z_{n-1} P_{n-2}\left(\frac{z_{1}}{\alpha_{1} \beta_{1}}, \ldots, \frac{z_{n-2}}{\alpha_{n-2} \beta_{n-2}}\right)+A \alpha_{n-1} P_{n-2}\left(\frac{\overline{\beta_{1}} z_{1}}{\alpha_{1}}, \ldots, \frac{\overline{\beta_{n-2} z_{n-2}}}{\alpha_{n-2}}\right)} .
$$

The condition $\left|z_{n}\right|=1$ for all $z_{n-1},\left|z_{n-1}\right|=1$, is equivalent, using Lemma 1, to:

$$
\left\{\begin{aligned}
\overline{\alpha_{n-1}} B P_{n-2}\left(\overline{\alpha_{1}} z_{1} / \beta_{1}, \ldots, \overline{\alpha_{n-2}} z_{n-2} / \beta_{n-2}\right) & =\kappa a \\
P_{n-2}\left(\overline{\alpha_{1}} \overline{\beta_{1}} z_{1}, \ldots, \overline{\alpha_{n-2}} \overline{\beta_{n-2}} z_{n-2}\right) & =\kappa b \\
A B P_{n-2}\left(z_{1} / \alpha_{1} \beta_{1}, \ldots, z_{n-2} / \alpha_{n-2} \beta_{n-2}\right) & =\bar{b} \\
A \alpha_{n-1} P_{n-2}\left(\overline{\beta_{1}} z_{1} / \alpha_{1}, \ldots, \overline{\beta_{n-2}} z_{n-2} / \alpha_{n-2}\right) & =\bar{a} .
\end{aligned}\right.
$$

This gives the equations

$$
\begin{aligned}
& \overline{P_{n-2}\left(z_{1} / \alpha_{1} \beta_{1}, \ldots, z_{n-2} / \alpha_{n-2} \beta_{n-2}\right)}=\frac{1}{\kappa \overline{A B}} P_{n-2}\left(\overline{\alpha_{1}} \overline{\beta_{1}} z_{1}, \ldots, \overline{\alpha_{n-2}} \overline{\beta_{n-2}} z_{n-2}\right), \\
& \overline{P_{n-2}\left(\overline{\beta_{1}} z_{1} / \alpha_{1}, \ldots, \overline{\beta_{n-2}} z_{n-2} / \alpha_{n-2}\right)}=\frac{B}{\kappa \bar{A}} P_{n-2}\left(\overline{\alpha_{1}} z_{1} / \beta_{1}, \ldots, \overline{\alpha_{n-2}} z_{n-2} / \beta_{n-2}\right) .
\end{aligned}
$$

We denote by $\bar{P}$ the polynomial with conjugate coefficients. Thus we get, since $\left|z_{j}\right|=1$,

$$
\begin{aligned}
\overline{P_{n-2}}\left(1 / \overline{\alpha_{1}} \overline{\beta_{1}} z_{1}, \ldots, 1 / \overline{\alpha_{n-2}} \overline{\beta_{n-2}} z_{n-2}\right) & =\frac{1}{\kappa \overline{A B}} P_{n-2}\left(\overline{\alpha_{1}} \overline{\beta_{1}} z_{1}, \ldots, \overline{\alpha_{n-2}} \overline{\beta_{n-2}} z_{n-2}\right), \\
\overline{P_{n-2}}\left(\beta_{1} / \overline{\alpha_{1}} z_{1}, \ldots, \beta_{n-2} / \overline{\alpha_{n-2}} z_{n-2}\right) & =\frac{B}{\kappa \bar{A}} P_{n-2}\left(\overline{\alpha_{1}} z_{1} / \beta_{1}, \ldots, \overline{\alpha_{n-2}} z_{n-2} / \beta_{n-2}\right) .
\end{aligned}
$$

Both formulae follow, with $\kappa=z_{1} \cdots z_{n-2}$ (which satisfies $|\kappa|=1$ ), from the relation

$$
\overline{P_{n}}\left(z_{1}, \ldots, z_{n}\right)=z_{1} \cdots z_{n} P_{n}\left(1 / z_{1}, \ldots, 1 / z_{n}\right),
$$

written for $n-2$.

Formula (7) itself is satisfied by $P_{1}$, and (as one checks easily) follows from the induction relation (5). This finishes the proof of Proposition 2. We observe that formula (7) may be considered as the complex analogue of (4).

We may now give an explicit formula for each $P_{n}\left(z_{1}, \ldots, z_{n}\right)$. Let us construct the first ones, using the induction formula (5):

$$
\begin{aligned}
P_{1}\left(z_{1}\right)= & A_{1} z_{1}+\overline{A_{1}}, \\
P_{2}\left(z_{1}, z_{2}\right)= & \overline{A_{1}}+A_{1} \overline{A_{2,1}} z_{1}+\overline{A_{1}} A_{2,1} z_{2}+A_{1} z_{1} z_{2}, \\
P_{3}\left(z_{1}, z_{2}, z_{3}\right)= & \overline{A_{1}}+A_{1} \overline{A_{2,1}} \overline{A_{3,1}} z_{1}+\overline{A_{1}} A_{2,1} \overline{A_{3,2} z_{2}}+\overline{A_{1}} A_{3,1} A_{3,2} z_{3} \\
& +A_{1} \overline{A_{3,1}} \overline{A_{3,2}} z_{1} z_{2}+A_{1} \overline{A_{2,1}} A_{3,2} z_{1} z_{3}+\overline{A_{1}} A_{2,1} A_{3,1} z_{2} z_{3}+A_{1} z_{1} z_{2} z_{3} .
\end{aligned}
$$


At that stage, the distribution of conjugates $\overline{A_{i, j}}$ looks unclear. Since the induction (5) reduces to (3) when all coefficients are real, we have obviously

$$
P_{n}\left(z_{1}, \ldots, z_{n}\right)=\sum_{S} z^{S} \prod_{i \in S} \prod_{j \in S^{\prime}} A_{i, j}^{*}
$$

where $A_{i, j}^{*}$ is either $A_{i, j}$ or $\overline{A_{i, j}}$ (and $A_{j, i}^{*}=A_{i, j}^{*}$ ). The choice is made according to the following rule:

- if $A_{1}^{*}$ is in a term which contains $z_{1}$, it will be $A_{1}$, otherwise $\overline{A_{1}}$,

- if $A_{2,1}^{*}$ is in a term which contains $z_{2}$, it will be $A_{2,1}$, otherwise $\overline{A_{2,1}}$,

- if $A_{3,1}^{*}, A_{3,2}^{*}$ are in a term which contains $z_{3}$, they will be $A_{3,1}, A_{3,2}$, otherwise $\overline{A_{3,1}}, \overline{A_{3,2}}$, and so on.

So for instance the term $A_{1}^{*} A_{2,1}^{*} A_{3,1}^{*} z_{2} z_{3}$, in $P_{3}$, will be $\overline{A_{1}} A_{2,1} A_{3,1} z_{2} z_{3}$ (no $z_{1}$, one $z_{2}$, one $z_{3}$ ).

This rule is clear from the induction procedure: if I want to construct, say, $P_{8}$, the coefficient $A_{4,3}$, for instance, will be met in the construction of $P_{4}$, and it appears as $A_{4,3}$ with $z_{4}$, and as $\overline{A_{4,3}}$ without $z_{4}$, and these properties will not be modified in the further stages of the construction.

Until now, as we already said, no assumption has been made on the $A_{i, j}$ 's, and the sole implication

$$
\left\{P_{n}\left(z_{1}, \ldots, z_{n}\right)=0 \text { and }\left|z_{1}\right|=\cdots=\left|z_{n-1}\right|=1 \text { implies }\left|z_{n}\right|=1\right\}
$$

does not, in itself, guarantee that the contracted polynomial $P_{n}(z)$ has all its roots on the unit circle. Indeed, take $P_{2}$ with $A_{1}=1, A_{2,1}=2: P_{2}\left(z_{1}, z_{2}\right)=1+2 z_{1}+$ $2 z_{2}+z_{1} z_{2}$, but $P_{2}(z)=1+4 z+z^{2}$ does not have its zeros on the unit circle. The assumption $\left|A_{i, j}\right| \leqq 1$ will ensure this conclusion.

Proposition 3. Let $\left(A_{i, j}\right)$ be a family of complex numbers with $\left|A_{i, j}\right| \leqq 1$. Then the complex polynomials $P_{n}\left(z_{1}, \ldots, z_{n}\right)$ defined by (5) or (8) satisfy

$$
\text { If } P_{n}\left(z_{1}, \ldots, z_{n}\right)=0 \text { and }\left|z_{1}\right| \leqq 1, \ldots,\left|z_{n-1}\right| \leqq 1 \text {, then }\left|z_{n}\right| \geqq 1 .
$$

Proof. It is made by induction and follows the lines given by Ruelle. The first step is for $n=2$. If $P_{2}\left(z_{1}, z_{2}\right)=0$,

$$
z_{2}=-\frac{A_{1} \overline{A_{2,1}} z_{1}+\overline{A_{1}}}{A_{1} z_{1}+\overline{A_{1}} A_{2,1}}=-\frac{\overline{A_{1}}}{A_{1}} \frac{\frac{A_{1}}{\overline{A_{1}}} \overline{A_{2,1}} z_{1}+1}{z_{1}+\overline{\overline{A_{1}}} A_{2,1}} .
$$

The last fraction is of the form $\frac{\bar{\alpha} z_{1}+1}{z_{1}+\alpha}$, with $|\alpha| \leqq 1$, so it is the inverse of a Blaschke term and satisfies $\left|z_{2}\right| \geqq 1$ if $\left|z_{1}\right| \leqq 1$.

Assume now the property has been proved until $n-1$ and assume it is false for $n$ : there are complex numbers $z_{1}^{0}, \ldots, z_{n}^{0}$, with

$$
\left|z_{1}^{0}\right| \leqq 1, \ldots,\left|z_{n-1}^{0}\right| \leqq 1,\left|z_{n}^{0}\right|<1, \quad P_{n}\left(z_{1}^{0}, \ldots, z_{n}^{0}\right)=0
$$

We can assume that in $P_{n}$ all $A_{i, j}$ 's satisfy $\left|A_{i, j}\right|<1$, for the limit cases follow by continuity.

Fix $z_{1}=z_{1}^{0}, \ldots, z_{n-2}=z_{n-2}^{0}$, and consider the condition

$$
P_{n}\left(z_{1}^{0}, \ldots, z_{n-2}^{0}, z_{n-1}, z_{n}\right)=0 \text {. }
$$


It determines a correspondence between $z_{n}$ and $z_{n-1}$ of the form

$$
z_{n}=\frac{a z_{n-1}+b}{c z_{n-1}+d}=\varphi\left(z_{n-1}\right)
$$

(the explicit form is given in (6)).

Let us call $z_{n-1}^{1}$ the point $z_{n-1}$ such that $\varphi\left(z_{n-1}^{1}\right)=0$. By (5), if $z_{n}=0$,

$$
P_{n-1}\left(\overline{A_{n, 1}} z_{1}^{0}, \ldots, \overline{A_{n, n-2}} z_{n-2}^{0}, \overline{A_{n, n-1}} z_{n-1}^{1}\right)=0 .
$$

Since $\left|\overline{A_{n, 1}} z_{1}^{0}\right|<\left|z_{1}^{0}\right| \leqq 1, \ldots,\left|\overline{A_{n, n-2}} z_{n-2}^{0}\right|<\left|z_{n-2}^{0}\right| \leqq 1$, this implies, by the induction hypothesis, that $\left|\overline{A_{n, n-1}} z_{n-1}^{1}\right| \geqq 1$, and so $\left|z_{n-1}^{1}\right|>1$.

So the function $\varphi$ maps $z_{n-1}^{0}$, with $\left|z_{n-1}^{0}\right| \leqq 1$ to $z_{n}^{0}$, with $\left|z_{n}^{0}\right|<1$, and maps $z_{n-1}^{1}$, with $\left|z_{n-1}^{1}\right|>1$, to 0 . We conclude that there is an intermediate point, say $z_{n-1}^{\prime}$, with $\left|z_{n-1}^{\prime}\right|=1$, such that the associated $z_{n}$, say $z_{n}^{\prime}$, satisfies $\left|z_{n}^{\prime}\right|<1$. This means, in fact, that we can take $\left|z_{n-1}\right|=1$ in (10).

Now, all variables play a similar role (this is clear from the form (8)), so we can consider the correspondence between $z_{n}$ and $z_{n-2}$, which will be determined by a formula similar to (6). The same argument shows that we can take $\left|z_{n-2}\right|=1$ in (10), and so on: we get finally $\left|z_{1}\right|=1, \ldots,\left|z_{n-1}\right|=1,\left|z_{n}\right|<1$, which contradicts Proposition 2 and finishes the proof.

\section{A Simple Proof in a Special Case}

There is a case in which a direct proof can be given, of the fact that the polynomials $P_{n}(z)$ have all their zeros on the unit circle, without using the many-variable $P_{n}\left(z_{1}, \ldots, z_{n}\right)$. Indeed, if for each $n$ all $A_{n, j}$ are equal, say to $\alpha_{n}$, the induction relations (5) and (7) give relations simply on the $P_{n}(z)$, which are

$$
\begin{aligned}
& P_{n}(z)=z \alpha_{n}^{n-1} P_{n-1}\left(\frac{z}{\alpha_{n}}\right)+P_{n-1}\left(\overline{\alpha_{n}} z\right), \\
& \overline{P_{n}}(z)=z^{n} P_{n}(1 / z) .
\end{aligned}
$$

Assume you have proved by induction that all zeros of $P_{n-1}$ are of modulus 1 , and write

$$
P_{n-1}=A_{1}\left(z-\gamma_{1}\right) \cdots\left(z-\gamma_{n-1}\right) .
$$

So, by (11),

$$
P_{n}(z)=z \alpha_{n}^{n-1}\left(A_{1}\left(\frac{z}{\alpha_{n}}-\gamma_{1}\right) \cdots\left(\frac{z}{\alpha_{n}}-\gamma_{n-1}\right)\right)+A_{1}\left(\overline{\alpha_{n}} z-\gamma_{1}\right) \cdots\left(\overline{\alpha_{n}} z-\gamma_{n-1}\right),
$$

and the condition $P_{n}=0$ reads

$$
z=-\frac{\overline{\alpha_{n}} z-\gamma_{1}}{z-\alpha_{n} \gamma_{1}} \cdots \frac{\overline{\alpha_{n}} z-\gamma_{n-1}}{z-\alpha_{n} \gamma_{n-1}}
$$

But each term $\frac{\overline{\alpha_{n}} z-\gamma_{J}}{z-\alpha_{n} \gamma_{J}}$ is the inverse of a Blaschke term, so has modulus $\geqq 1$ inside the unit disk. So equality (12) is possible only for $z$ with $|z|=1$. 


\section{Where are the Roots of the Complex Lee-Yang Polynomials?}

Of course, by what we saw previously, they are on the unit circle. But are there further restrictions, as it was the case with the real polynomials? The answer is no:

Proposition 4. For any choice of $z_{1}, \ldots, z_{n}$, with $\left|z_{1}\right|=\cdots=\left|z_{n}\right|=1$, there is a complex Lee-Yang polynomial $P_{n}$, the zeros of which are exactly $z_{1}, \ldots, z_{n}$.

Proof. The proof will be by induction on $n$, and, for all $n$, the $P_{n}$ we build will satisfy (as in Sect. 3 above)

$$
A_{n, 1}=\cdots=A_{n, n-1}=\alpha_{n},
$$

with now $\left|\alpha_{n}\right|=1$.

For $n=1$, the statement is clear. Assume the proposition holds until $n-1$. Then, Eq. (11) reads

$$
P_{n}(z)=\left(1+\alpha_{n}^{n-1} z\right) P_{n-1}\left(\overline{\alpha_{n}} z\right) .
$$

So, given $z_{1}, \ldots, z_{n}$ with $\left|z_{j}\right|=1$, we choose first $\alpha_{n}$ so that $\left|\alpha_{n}\right|=1$ and $-1 / \alpha_{n}^{n-1}=z_{n}$. Then, using the induction hypothesis, we choose $\alpha_{1}, \ldots, \alpha_{n-1}$ so that the zeros of $P_{n-1}(z)$ are precisely $\overline{\alpha_{n}} z_{1}, \ldots, \overline{\alpha_{n}} z_{n-1}$ : so the zeros of $P_{n-1}\left(\overline{\alpha_{n}} z\right)$ will be $z_{1}, \ldots, z_{n-1}$. This completes the proof of the proposition.

\section{A Class of Inner Functions in Several Variables}

The complex Lee and Yang polynomials, which we just introduced, will allow us to define a class of inner functions in several complex variables. Indeed, if $P_{n}=0,-1 / z_{n}$ satisfies $1 /\left|z_{n}\right|=1$ if $\left|z_{1}\right|=\cdots\left|z_{n-1}\right|=1$ and $1 /\left|z_{n}\right| \leqq 1$ if $\left|z_{1}\right| \leqq$ $1, \ldots,\left|z_{n-1}\right| \leqq 1$. This leads to the following proposition:

Proposition 5. Let $P_{n}\left(z_{1}, \ldots, z_{n}\right)$ be the complex Lee and Yang polynomials defined above. Let $\alpha_{1}, \ldots, \alpha_{n}$ be complex numbers with $\left|\alpha_{j}\right| \leqq 1$. The function

$$
f_{n}\left(z_{1}, \ldots, z_{n}\right)=\frac{\alpha_{1} \cdots \alpha_{n} P_{n}\left(z_{1} / \alpha_{1}, \ldots, z_{n} / \alpha_{n}\right)}{P_{n}\left(\overline{\alpha_{1}} z_{1}, \ldots, \overline{\alpha_{n}} z_{n}\right)}
$$

is an inner function: it is analytic in $D \times \cdots \times D$ and satisfies

$$
\begin{array}{ll}
\left|f_{n}\left(z_{1}, \ldots, z_{n}\right)\right| \leqq 1 & \text { if }\left|z_{1}\right| \leqq 1, \ldots,\left|z_{n}\right| \leqq 1 \\
\left|f_{n}\left(z_{1}, \ldots, z_{n}\right)\right|=1 & \text { if }\left|z_{1}\right|=\cdots=\left|z_{n}\right|=1 .
\end{array}
$$

Proof. This is just the expression of $-1 / z_{n+1}$ when $P_{n+1}=0$, where we have set $\alpha_{1}=A_{n+1,1}, \ldots \alpha_{n}=A_{n+1, n}$.

For $n=1$, we get

$$
f_{1}(z)=\frac{A z+\alpha \bar{A}}{A \bar{\alpha} z+\bar{A}}=\frac{A}{\bar{A}} \frac{z+\alpha \frac{\bar{A}}{A}}{1+\frac{A}{\bar{A}} \bar{\alpha} z},
$$


which is just a Blaschke term. For $n=2$, we get

$$
f_{2}\left(z_{1}, z_{2}\right)=\frac{A_{1} z_{1} z_{2}+\overline{A_{1}} A_{2,1} \alpha_{1} z_{2}+A_{1} \overline{A_{2,1}} \alpha_{2} z_{1}+\alpha_{1} \alpha_{2} \overline{A_{1}}}{A_{1} \overline{\alpha_{1}} \overline{\alpha_{2}} z_{1} z_{2}+\overline{A_{1}} A_{2,1} \overline{\alpha_{2}} z_{2}+A_{1} \overline{A_{2,1}} \overline{\alpha_{1}} z_{1}+\overline{A_{1}}}
$$

and so on. The $n^{\text {th }}$ function depends on $1+\frac{n(n+1)}{2}$ complex parameters. We observe that a zero $\left(z_{1}, \ldots, z_{n}\right)$ cannot be too close to $(0, \ldots, 0)$ : if $\left|z_{1}\right| \leqq\left|\alpha_{1}\right|, \ldots,\left|z_{n-1}\right| \leqq$ $\left|\alpha_{n-1}\right|$, then $\left|z_{n}\right| \geqq\left|\alpha_{n}\right|$.

\section{References}

1. Beauzamy, B.: Finding the roots of polynomial equations: An algorithm with linear command. To appear

2. Ruelle, D.: Statistical mechanics: Rigorous results. New York: Benjamin, 1969

3. Ruelle, D.: Is our mathematics natural? The case of equilibrium statistical mechanics. Bull. A.M.S., 19-1, 259-268 (1988)

4. Walsh, J.: On the location of the roots of certain types of polynomials. Trans. A.M.S. 24, 163-180 ((October) 1922)

Communicated by J.L. Lebowitz 